\title{
Haemoperfused liver as an ex vivo model for organ invasion of Candida albicans
}

\author{
Correspondence \\ Bernhard Hube \\ bernard.hube@hki-jena.de
}

Received 2 June 2006

Accepted 13 October 2006

\author{
Sascha Thewes, ${ }^{1}$ Hilde-Kristin Reed, ${ }^{2}$ Christian Grosse-Siestrup, ${ }^{2}$ \\ David A. Groneberg, ${ }^{2}$ Michael Meissler, ${ }^{2}$ Martin Schaller ${ }^{3}$ \\ and Bernhard Hube ${ }^{1} \dagger$ \\ ${ }^{1}$ Robert Koch Institute, Nordufer 20, 13353 Berlin, Germany \\ ${ }^{2}$ Department of Comparative Medicine and Experimental Animal Science, Charite School of \\ Medicine, 13353 Berlin, Germany \\ ${ }^{3}$ Institute of Dermatology, Eberhard Karls University, 72076 Tübingen, Germany
}

\section{INTRODUCTION}

In hospitals, Candida albicans is the leading pathogen causing disseminated fungal infections, but may also cause other life-threatening infections. For example, C. albicans causes intra-abdominal mycoses and invasive fungal infections after liver transplantation, with mortality rates of up to $60 \%$ (Boos et al., 2005; Fischer \& Sterneck, 2005). Furthermore, C. albicans may cause peritonitis during continuous ambulatory peritoneal dialysis (Michel et al., 1994). During these infections, the fungus colonizes the surface of parenchymal organs and penetrates into the tissue from the surface, but not from the bloodstream.

The most commonly used infection model for C. albicans is murine tail-vein injection for haematogenously disseminated candidosis. However, depending on the size of the inoculum of fungal cells, the outcome of such infections varies (Odds et al., 2000). Furthermore, these models are of limited use in studying the invasion properties of $C$. albicans, as direct injection of the fungal cells into the bloodstream means that the cells do not have to invade actively through host tissue to reach the bloodstream. For this reason, other infection models such as epithelial monolayers, reconstituted human epithelium, chick

tPresent address: Chair and Professor of Microbial Pathogenicity, Friedrich Schiller University Jena, Head of Department of Microbial Pathogenicity, Leibniz Institute for Natural Product Research and Infection Biology - Hans Knoell Institute Jena (HKI), Beutenbergstrabe 11a, 07745 Jena, Germany.

Abbreviations: ALT, alanine aminotransferase; AST, aspartate aminotransferase; i.p., intraperitoneal; p.i., post-infection. chorioallantoic membrane and intraperitoneal (i.p.) infections have been established to study invasion of $C$. albicans (Farrell et al., 1983; Gow et al., 2003; Kretschmar et al., 1999a, b; Schaller et al., 1998). In addition to qualitative histological examination, parameters such as lactate dehydrogenase and alanine aminotransferase (ALT) levels can be analysed to quantify tissue damage and invasion in these models (Kretschmar et al., 1999a; Schaller et al., 1998).

One possible way of avoiding experimentation on living animals is to use models based on ex vivo perfused organs. Perfused organs have been used for the investigation of many different physiological, pharmacological and toxicological aspects (Gordon et al., 1972; Groneberg et al., 2002). Following multi-organ harvesting or using organs from slaughtered animals, perfusion has the further advantage that it contributes to the ' $3 \mathrm{R}$ ' concept (refinement, reduction, replacement) of animal experimentation by reducing animal usage for research purposes (Grosse-Siestrup et al., 2002a, b). Studies in the mid-1970s used perfused livers from mice as infection models for bacteria and C. albicans (Moon et al., 1975; Sawyer et al., 1976) to investigate clearance of the micro-organisms from the bloodstream. The aim of our study was to establish an ex vivo haemoperfused liver from pigs as a model to study invasion of $C$. albicans.

\section{METHODS}

Strains and media. C. albicans strains SC5314 (Gillum et al., 1984) and ATCC 10231 (Kretschmar et al., 1999a) were incubated for $18 \mathrm{~h}$ in Sabouraud medium containing $2 \%$ glucose. Before 
infection, cells were harvested by centrifugation, washed three times with PBS and resuspended in PBS to a final concentration of $2 \times 10^{9}$ cells $\mathrm{ml}^{-1}$.

Animals. White German Landrace pigs (five male, three female; weight $42 \pm 13 \mathrm{~kg}$ ) were used with the approval of the official veterinary institutions. Animals used in this study received humane care and study protocols were in accordance with the guidelines of the German authorities for the care and use of laboratory animals. Animals were killed by final exsanguination during the bloodharvesting procedure.

Organ harvesting. All organs were harvested under general anaesthesia as described previously (Nagel et al., 2005). Directly after preparation of the blood vessels and after blood harvesting, the liver was flushed via the aorta with 41 pre-warmed $\left(37^{\circ} \mathrm{C}\right)$ electrolyte infusion solution 153 (Berlin-Chemie) to wash residual blood from the organ. The liver was removed and the portal vein, hepatic artery, vena cava and bile duct were cannulated. The cannulated organ was used directly for perfusion. Initial liver weight was determined to be $939 \pm 208 \mathrm{~g}$

Blood harvesting. Prior to blood harvesting, one bolus of heparin $(10000 \mathrm{U})$ was administered to the animals. Collection of blood was performed by cannulation of the cervical vessels on one side immediately before organ harvesting. The collected blood (1.5 l) was further treated with heparin $\left(10000 \mathrm{U}^{-1}\right)$ and an antibiotic (Augmentan, $1.2 \mathrm{~g} \mathrm{l}^{-1}$; GlaxoSmithKline). Just before connection of the organ, blood was added to the perfusion circuit and pre-warmed to $37^{\circ} \mathrm{C}$.

Perfusion. To set up our perfusion model, four livers were perfused for $3 \mathrm{~h}$ without the application of fungal cells. Perfusion was carried out as described previously (Grosse-Siestrup et al., 2002b, 2004) with modifications (Fig. 1). The blood for the hepatic artery came directly from the modules for oxygenation and dialysis (oxygenrich). For the portal vein, a mixture of oxygen-rich (from the dialysis module) and oxygen-poor (venous) blood was chosen to obtain a relatively physiological situation of oxygen for the liver. Roller pumps were used for the dialysis circuit as well as for the blood circuit. The connected liver was put into a sterile plastic bag to create a humid atmosphere and placed on a waterbed kept at $37^{\circ} \mathrm{C}$. Perfusion parameters were adjusted and maintained as described previously (Grosse-Siestrup et al., 2002b, 2004). During perfusion, samples of blood and dialysate were taken periodically for standard clinical laboratory analysis, as described previously (Grosse-Siestrup et al., 2002b, 2004). This included determination of the concentrations of the marker enzymes ALT and aspartate aminotransferase (AST) in the blood. The amount of bile production was measured at the indicated time points.

Application of Candida cells. After establishing the model, four livers were infected with C. albicans and perfused for $12 \mathrm{~h}$ (see above). Cells were applied to the liver surface following a protocol modified from that of Kvaal et al. (1999). Briefly, a sterile cotton patch $\left(0.5 \mathrm{~cm}^{2}\right)$ was inoculated with $10 \mu \mathrm{l}$ C. albicans suspension $\left(2 \times 10^{7}\right.$ cells $)$, applied to the surface and covered with a sterile plastic patch $\left(1 \mathrm{~cm}^{2}\right)$ to protect the fungal cells and prevent a freeoxygen exchange with the environment (microaerophilic to anaerobic conditions). For each strain and each time point, a single cotton patch was used and different strains and different time points were investigated on a single organ.

Histological examination. For histological examination of the invasion process, the fungal patches were removed from the surface at the indicated time points and the underlying region of the liver with adhered and invaded cells was cut out with a sterile scalpel. As a control, a piece of uninfected liver was cut out at the end of the perfusion. The liver pieces were fixed immediately in $2.5 \%$ glutaraldehyde with $2 \%$ paraformaldehyde in PBS (pH 7.4) and stored
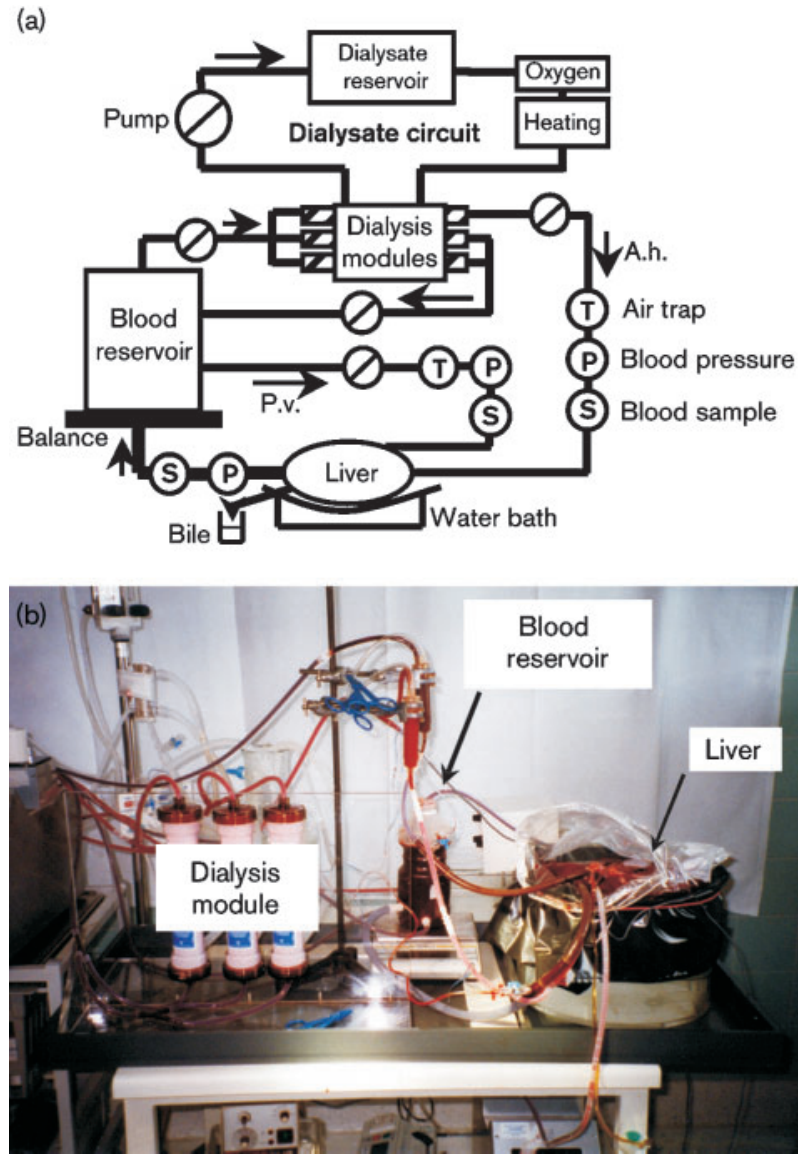

Fig. 1. Liver perfusion set-up. (a) Schematic drawing of the blood and dialysate circuit. (b) Photograph of the laboratory set-up. A.h., arteria hepatica circuit; P.v., portal vein circuit.

at room temperature until further use. Embedding, cutting and staining were carried out as described previously (Schaller et al., 1999).

Statistical analysis. All data were analysed using Microsoft Excel. If not indicated otherwise, data are presented as means \pm SD. Significance was calculated using Student's $t$-test.

\section{RESULTS AND DISCUSSION}

Several studies have described the extracorporeal haemoperfused liver as a suitable model to study, for example, hepatotoxicity (Groneberg et al., 2002). Other protocols have shown that a liver can be perfused successfully for up to $72 \mathrm{~h}$ (Butler et al., 2002). However, none of the described models have used the perfused organ to study interaction with pathogenic micro-organisms. Here, we describe for the first time the successful infection of extracorporeal haemoperfused porcine liver with the human fungal pathogen C. albicans.

\section{Perfusion set-up}

To keep the set-up of our model as simple as possible, we developed a protocol based only on general medical 
equipment (e.g. roller pumps instead of artificial hearts). To supply the liver with blood with an oxygen level similar to physiological conditions, we mixed the oxygen-poor ('venous') blood in our blood reservoir with oxygen-rich ('arterial') blood and applied this mixture to the organ via the portal vein (in parallel with oxygen-rich blood via the hepatic artery; Fig. 1). With this set-up, we were able to keep the liver functional (see below). In fact, histological examination after $12 \mathrm{~h}$ of perfusion showed no lesions and showed that the surface of the organ was intact (see Fig. 3a). Therefore, our set-up was comparable with previously perfused organs (Butler et al., 2002; Nagel et al., 2005).

\section{Perfusion parameters}

For functional analysis of the perfused liver, we chose parameters such as bile production, oxygen consumption and liver weight gain, and measured the blood concentration of the marker enzymes ALT and AST to quantify liver damage. ALT and AST are marker enzymes of the liver and an increased level of each of these enzymes in the blood is an established indicator of liver damage (Giannini et al., 2005).

As shown in Table 1, bile was constantly produced over the whole perfusion time in infected livers. Similarly, oxygen was consumed by the infected organs with a slight decrease over time. These data were similar to those obtained from uninfected organs (data not shown) and to other perfusion systems (Butler et al., 2002; Grosse-Siestrup et al., 2002b; Nagel et al., 2005). We observed only a moderate weight gain of the organs after $12 \mathrm{~h}$ of perfusion compared with the $3 \mathrm{~h}$ perfused livers (Table 1). This gain was possibly due to oedema caused by post-ischaemic injury (Nagel et al., 2005).

For the determination of ALT and AST levels, we compared data from uninfected livers with data from the first $3 \mathrm{~h}$ of the infected organ. We also determined the enzyme levels of the infected livers after $3 \mathrm{~h}$; however, at this time point we began to take samples by cutting out liver pieces for histological examination. Therefore, ALT and AST concentrations were increased in the blood due to the cutting process (data not

Table 1. Bile production, oxygen consumption and liver weight gain during $12 \mathrm{~h}$ of perfusion $(n=4)$

\begin{tabular}{|cccc|}
\hline $\begin{array}{c}\text { Time } \\
(\mathbf{m i n})\end{array}$ & $\begin{array}{c}\text { Bile production } \\
\left(\mathbf{m l ~ h}^{-\mathbf{1}}\right)\end{array}$ & $\begin{array}{c}\text { Oxygen consumption } \\
\left(\boldsymbol{\mu} \mathbf{m o l} \mathbf{~ m i n}^{-\mathbf{1}} \text { per } \mathbf{1 0 0} \mathbf{~ g}\right)\end{array}$ & $\begin{array}{c}\text { Liver weight } \\
\text { gain }(\%)^{*}\end{array}$ \\
\hline 60 & $2.55 \pm 3.88$ & $81.34 \pm 16.99$ & $\mathrm{NA}$ \\
180 & $3.13 \pm 0.75$ & $87.12 \pm 11.56$ & $11.65 \pm 11.03 \dagger$ \\
300 & $1.99 \pm 1.20$ & $81.91 \pm 12.30$ & $\mathrm{NA}$ \\
420 & $1.43 \pm 1.13$ & $68.26 \pm 27.78$ & $\mathrm{NA}$ \\
540 & $2.70 \pm 1.61$ & $77.97 \pm 22.06$ & $\mathrm{NA}$ \\
720 & $2.35 \pm 0.85$ & $66.58 \pm 24.83$ & $16.98 \pm 21.85$ \\
\hline
\end{tabular}

${ }^{\star}$ NA, Not applicable.

$\dagger$ Liver weight gain of the four livers used to set up this model.

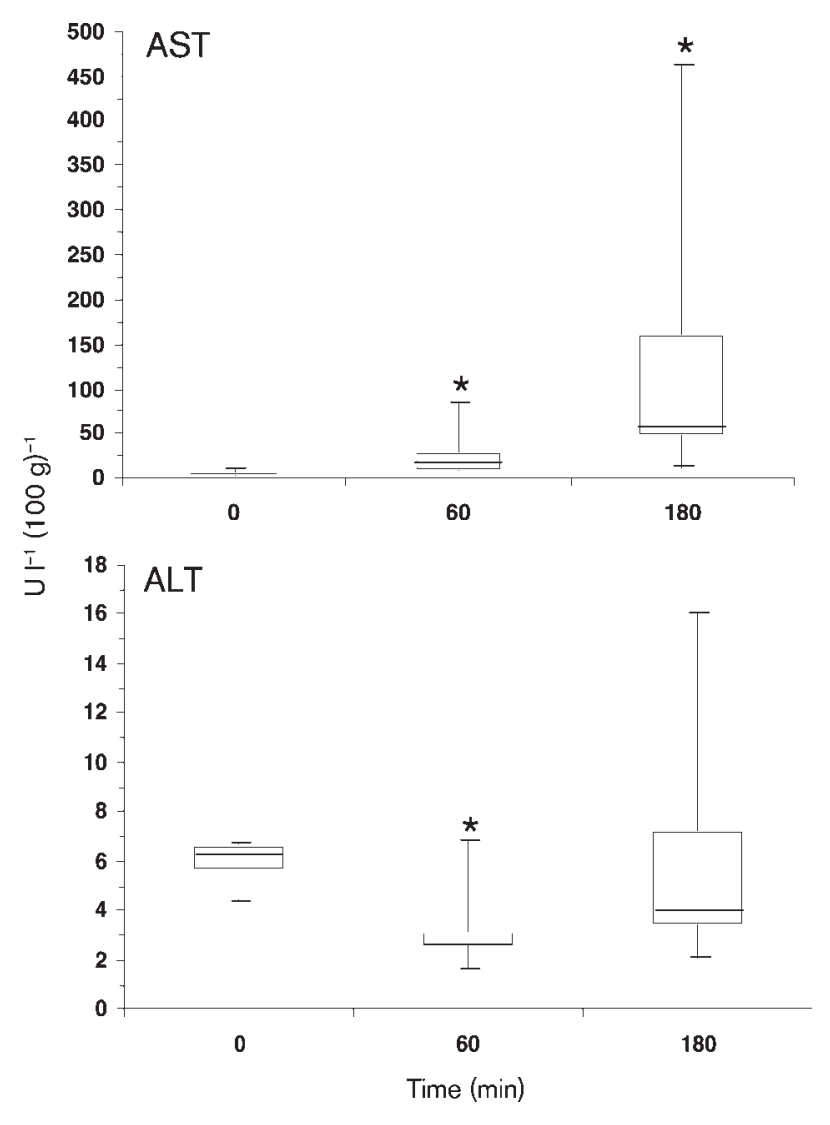

Fig. 2. Box plot of AST and ALT concentrations in the blood from eight different perfusions. *, Significant difference compared with the initial value $(P<0.05)$.

shown). As shown in Fig. 2, the level of AST increased slightly but significantly during the first $3 \mathrm{~h}$, whereas the ALT level was relatively constant and in the normal porcine range (Butler et al., 2002). The increase in AST level could be explained again by post-ischaemic injury. No difference was detected between infected and uninfected livers within the first $3 \mathrm{~h}$ (not shown), showing that the damage of the organ due to the infection process with Candida cells is negligible in the early stages of invasion. This finding is in agreement with the in vivo situation where an increase in enzyme levels after i.p. infection can only be measured at later time points (after 6 h in mice; Kretschmar et al., 1999a).

\section{Comparison of invasive and non-invasive strains}

To study the invasion of $C$. albicans into the perfused pig liver, we used two different approaches: the first approach was a comparison of an invasive strain (SC5314) and a noninvasive strain (ATCC 10231; Kretschmar et al., 1999a, b). The second approach was a time course of invasion of strain SC5314. Based on the results obtained during in vivo infection of mice (Felk et al., 2002; Kretschmar et al., 1999a), we took samples for histological examination at time points of 3 (not shown), 5, 7 and 10 h post-infection (p.i.) (Fig. 3). 


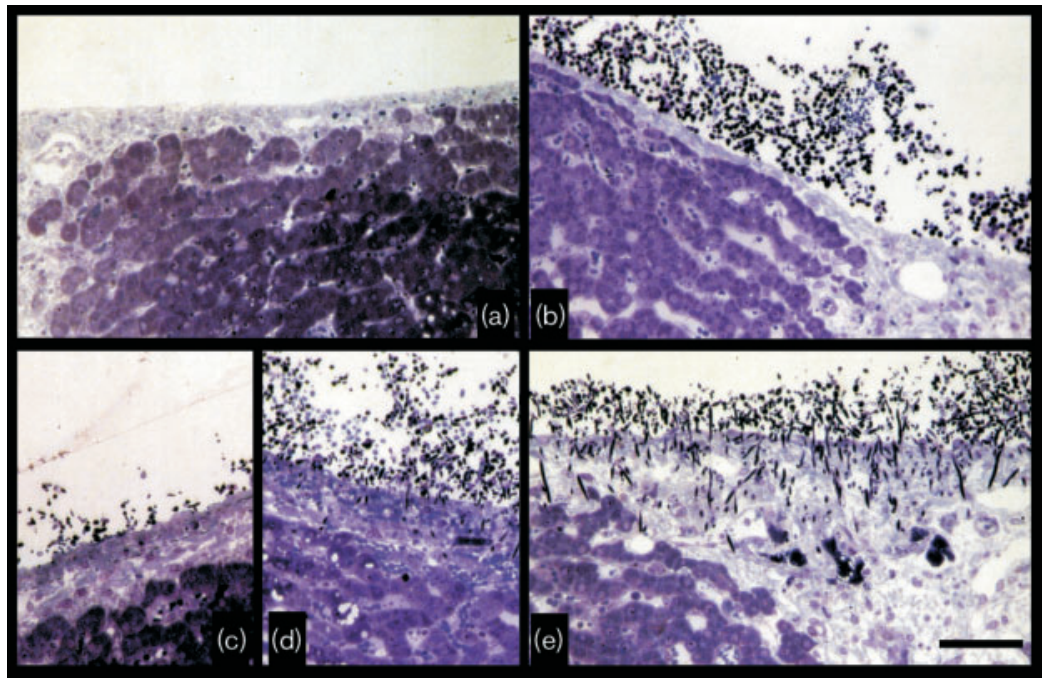

Fig. 3. Representative pictures of uninfected liver after $12 \mathrm{~h}$ of perfusion (a) and of liver infected with $C$. albicans (b-e). Strain ATCC 10231 showed no invasion after $10 \mathrm{~h}$ of perfusion (b); strain SC5314 showed no invasion after $5 \mathrm{~h}$ (c) but started to invade after $7 \mathrm{~h}(\mathrm{~d})$ and reached deeper hepatocytes after $10 \mathrm{~h}$ of perfusion (e). Bar, $25 \mu \mathrm{m}$.

As shown in Fig. 3, the highly virulent strain SC5314 had invaded deep into the perfused liver after $10 \mathrm{~h}$ (Fig. 3e), whereas strain ATCC 10231 was clearly non-invasive (Fig. 3b). The starting inoculum seemed not to play a role during local invasion, as lower cell numbers showed the same differences between the two strains (data not shown). This result is consistent with the findings in i.p.-infected mice (Kretschmar et al., 1999a, b). This non-invasive phenotype of ATCC 10231 is likely to be the main reason for the fact that this particular C. albicans strain is avirulent in all animal models tested so far (Balish \& Phillips, 1966; Phillips \& Balish, 1966; Schmidt \& Geschke, 1996).

\section{Time course of invasion}

As strain ATCC 10231 was non-invasive in our ex vivo perfused model (confirming that our model mimics the situation in vivo), we focused our analysis on the temporal progression of invasion of strain SC5314. As shown in Fig. 3(c), no invading cells of strain SC5314 were detected $5 \mathrm{~h}$ after infection of the surface of the liver. However, invasion was clearly visible $7 \mathrm{~h}$ after infection. Here, C. albicans cells had switched to the hyphal mode of growth and penetrated into the liver capsule (Fig. 3d). After $10 \mathrm{~h}$, hyphae had also reached the deeper hepatocytes (Fig. 3e). The invasion process over time was very similar to the observations made by Felk et al. (2002) and Kretschmar et al. (1999a) with i.p.-infected mice. The only difference observed in our model compared with in vivo infection was the fact that invasion into the capsule of the pig liver began later than in mice ( $\sim 4 \mathrm{~h}$ p.i. in mice vs $\sim 7 \mathrm{~h}$ p.i. in perfused livers). One reason for this difference might be the thicker organ capsule of the pig liver compared with the mouse liver.

\section{The perfused liver as a model to study invasion}

Several models to study interaction and/or invasion or transmigration of $C$. albicans through mammalian cells have been described in the literature. These models include in vitro monolayers to investigate the interaction of $C$. albicans with human umbilical vein endothelial cells (Filler et al., 1995) or brain microvascular endothelial cells (Jong et al., 2001), transmigration models for transmigration through endothelial layers (Zink et al., 1996) and intestinal cells (Weide \& Ernst, 1999), more sophisticated threedimensional models such as reconstituted human epithelium (Schaller et al., 1998) and in vivo models (Kretschmar et al., 1999a, b).

In our study, we showed that the ex vivo perfused liver is another suitable model to study invasion of $C$. albicans and possibly other microbes that invade human liver tissue. In contrast to the other models mentioned above, our invasion model has several advantages: (i) The large surface of the pig liver can be used to compare several strains or to investigate several time points of invasion with just one organ; this has the advantage that, for example, a mutant strain can be compared directly with the corresponding wild-type strain leading to a higher reproducibility compared with in vivo experiments where each strain or time point has to be tested in a separate animal. (ii) The ex vivo perfused liver represents the closest model of the in vivo situation (Groneberg et al., 2002). Although limited only to one infection site, this model includes almost the entire repertoire of immune cells and opens up the possibility of investigating interaction of C. albicans cells with host cells in an in vivo-like situation and analysing both the pathogen and the host simultaneously during infection. (iii) Finally, using perfused organs contributes to the ' $3 \mathrm{R}$ ' concept of animal experimentation (Grosse-Siestrup et al., 2002a, b) so that in future the number of animals required to study invasion of $C$. albicans could be reduced.

\section{ACKNOWLEDGEMENTS}

We would like to thank A. Mavor, Robert Koch Institute, for critical reading of the manuscript. We would also like to thank E. Januschke from the LMU Munich for technical assistance. This work has been 
supported by the Robert Koch Institute, the Charite School of Medicine and the EU projects 'Galar Fungail' (QLK2-2000-00795) and 'Galar Fungail 2' (MCRTN-2003-504148).

\section{REFERENCES}

Balish, E. \& Phillips, A. W. (1966). Growth, morphogenesis, and virulence of Candida albicans after oral inoculation in the germ-free and conventional chick. J Bacteriol 91, 1736-1743.

Boos, C., Kujath, P. \& Bruch, H.-P. (2005). Intra-abdominal mycoses. Mycoses 48 (Suppl. 1), 22-26 (in German).

Butler, A. J., Rees, M. A., Wight, D. G., Casey, N. D., Alexander, G., White, D. J. \& Friend, P. J. (2002). Successful extracorporeal porcine liver perfusion for $72 \mathrm{hr}$. Transplantation 73, 1212-1218.

Farrell, S. M., Hawkins, D. F. \& Ryder, T. A. (1983). Scanning electron microscope study of Candida albicans invasion of cultured human cervical epithelial cells. Sabouraudia 21, 251-254.

Felk, A., Kretschmar, M., Albrecht, A., Schaller, M., Beinhauer, S., Nichterlein, T., Sanglard, D., Korting, H. C., Schäfer, W. \& Hube, B. (2002). Candida albicans hyphal formation and the expression of the Efg1-regulated proteinases Sap4 to Sap6 are required for the invasion of parenchymal organs. Infect Immun 70, 3689-3700.

Filler, S. G., Swerdloff, J. N., Hobbs, C. \& Luckett, P. M. (1995). Penetration and damage of endothelial cells by Candida albicans. Infect Immun 63, 976-983.

Fischer, L. \& Sterneck, M. (2005). Invasive fungal infections in patients after liver transplantation. Mycoses 48 (Suppl. 1), 27-35 (in German).

Giannini, E. G., Testa, R. \& Savarino, V. (2005). Liver enzyme alteration: a guide for clinicians. CMAJ 172, 367-379.

Gillum, A. M., Tsay, E. Y. \& Kirsch, D. R. (1984). Isolation of the Candida albicans gene for orotidine- $5^{\prime}$-phosphate decarboxylase by complementation of S. cerevisiae ura3 and E. coli pyrF mutations. Mol Gen Genet 198, 179-182.

Gordon, E. M., Douglas, M. C., Jablonski, P., Owen, J. A., Sali, A. \& Watts, J. M. (1972). Gastroduodenal hormones and bile-secretion studies in the isolated perfused pig liver. Surgery 72, 708-721.

Gow, N. A., Knox, Y., Munro, C. A. \& Thompson, W. D. (2003). Infection of chick chorioallantoic membrane (CAM) as a model for invasive hyphal growth and pathogenesis of Candida albicans. Med Mycol 41, 331-338.

Groneberg, D. A., Grosse-Siestrup, C. \& Fischer, A. (2002). In vitro models to study hepatotoxicity. Toxicol Pathol 30, 394-399.

Grosse-Siestrup, C., Fehrenberg, C., von Baeyer, H. \& Groneberg, D. A. (2002a). Multiple-organ harvesting for models of isolated hemoperfused organs of slaughtered pigs. ALTEX 19, 9-13.

Grosse-Siestrup, C., Pfeffer, J., Unger, V., Nagel, S., Witt, C., Fischer, A. \& Groneberg, D. A. (2002b). Isolated hemoperfused slaughterhouse livers as a valid model to study hepatotoxicity. Toxicol Pathol 30, 749-754.

Grosse-Siestrup, C., Unger, V., Pfeffer, J., Dinh, Q. T., Nagel, S., Springer, J., Witt, C., Wussow, A. \& Groneberg, D. A. (2004).
Hepatotoxic effects of polidocanol in a model of autologously perfused porcine livers. Arch Toxicol 78, 697-705.

Jong, A. Y., Stins, M. F., Huang, S.-H., Chen, S. H. M. \& Kim, K. S. (2001). Traversal of Candida albicans across human blood-brain barrier in vitro. Infect Immun 69, 4536-4544.

Kretschmar, M., Bertsch, T., Goller, M., Schaller, M., Hof, H. \& Nichterlein, T. (1999a). Parameters for determination of Candida albicans virulence in murine peritonitis. Mycoses 42 (Suppl. 2), 19-24.

Kretschmar, M., Hube, B., Bertsch, T., Sanglard, D., Merker, R., Schröder, M., Hof, H. \& Nichterlein, T. (1999b). Germ tubes and proteinase activity contribute to virulence of Candida albicans in murine peritonitis. Infect Immun 67, 6637-6642.

Kvaal, C., Lachke, S. A., Srikantha, T., Daniels, K., McCoy, J. \& Soll, D. R. (1999). Misexpression of the opaque-phase-specific gene PEP1 (SAP1) in the white phase of Candida albicans confers increased virulence in a mouse model of cutaneous infection. Infect Immun 67, 6652-6662.

Michel, C., Courdavault, L., al Khayat, R., Viron, B., Roux, P. \& Mignon, F. (1994). Fungal peritonitis in patients on peritoneal dialysis. Am J Nephrol 14, 113-120.

Moon, R. J., Vrable, R. A. \& Broka, J. A. (1975). In situ separation of bacterial trapping and killing functions of the perfused liver. Infect Immun 12, 411-418.

Nagel, S., Hegemann, O., Groneberg, D. A. \& Grosse-Siestrup, C. (2005). An improved model of isolated hemoperfused porcine livers using pneumatically driven pulsating blood pumps. Toxicol Pathol 33, 434-440.

Odds, F. C., Van Nuffel, L. \& Gow, N. A. R. (2000). Survival in experimental Candida albicans infections depends on inoculum growth conditions as well as animal host. Microbiology 146, 1881-1889.

Phillips, A. W. \& Balish, E. (1966). Growth and invasiveness of Candida albicans in the germ-free and conventional mouse after oral challenge. Appl Microbiol 14, 737-741.

Sawyer, R. T., Moon, R. J. \& Beneke, E. S. (1976). Hepatic clearance of Candida albicans in rats. Infect Immun 14, 1348-1355.

Schaller, M., Schäfer, W., Korting, H. C. \& Hube, B. (1998). Differential expression of secreted aspartyl proteinases in a model of human oral candidosis and in patient samples from the oral cavity. Mol Microbiol 29, 605-615.

Schaller, M., Korting, H. C., Schäfer, W., Bastert, J., Chen, W. \& Hube, B. (1999). Secreted aspartic proteinase (Sap) activity contributes to tissue damage in a model of human oral candidosis. Mol Microbiol 34, 169-180.

Schmidt, A. \& Geschke, U. (1996). Comparative virulence of Candida albicans strains in CFW1 mice and Sprague-Dawley rats. Mycoses 39, 157-160.

Weide, M. R. \& Ernst, J. F. (1999). Caco-2 monolayer as a model for transepithelial migration of the fungal pathogen Candida albicans. Mycoses 42 (Suppl. 2), 61-67.

Zink, S., Nass, T., Rosen, P. \& Ernst, J. F. (1996). Migration of the fungal pathogen Candida albicans across endothelial monolayers. Infect Immun 64, 5085-5091. 